\title{
On the first Zagreb index and multiplicative Zagreb coindices of graphs
}

\section{Kinkar Ch. Das, Nihat Akgunes, Muge Togan, Aysun Yurttas, I. Naci Cangul, A. Sinan Cevik}

\begin{abstract}
For a (molecular) graph $G$ with vertex set $V(G)$ and edge set $E(G)$, the first Zagreb index of $G$ is defined as $M_{1}(G)=\sum_{v_{i} \in V(G)} d_{G}\left(v_{i}\right)^{2}$, where $d_{G}\left(v_{i}\right)$ is the degree of vertex $v_{i}$ in $G$. Recently Xu et al. introduced two graphical invariants $\overline{\prod_{1}}(G)=\prod_{v_{i} v_{j} \notin E(G)}\left(d_{G}\left(v_{i}\right)+d_{G}\left(v_{j}\right)\right)$ and $\overline{\prod_{2}}(G)=\prod_{v_{i} v_{j} \notin E(G)} d_{G}\left(v_{i}\right) d_{G}\left(v_{j}\right)$ named as first multiplicative Zagreb coindex and second multiplicative Zagreb coindex, respectively. The Narumi-Katayama index of a graph $G$, denoted by $N K(G)$, is equal to the product of the degrees of the vertices of $G$, that is, $N K(G)=$ $\prod_{i=1}^{n} d_{G}\left(v_{i}\right)$. The irregularity index $t(G)$ of $G$ is defined as the number of distinct terms in the degree sequence of $G$. In this paper, we give some lower and upper bounds on the first Zagreb index $M_{1}(G)$ of graphs and trees in terms of number of vertices, irregularity index, maximum degree, and characterize the extremal graphs. Moreover, we obtain some lower and upper bounds on the (first and second) multiplicative Zagreb coindices of graphs and characterize the extremal graphs. Finally, we present some relations between first Zagreb index and NarumiKatayama index, and (first and second) multiplicative Zagreb index and coindices of graphs.
\end{abstract}

Key Words: First Zagreb index, First and Second multiplicative Zagreb coindex, Narumi-Katayama index.

2010 Mathematics Subject Classification: Primary 05C07; Secondary $05 \mathrm{C} 12$

Received: 3.05.2014

Accepted: 2.10 .2014 


\section{Introduction}

We only consider finite, undirected and simple graphs throughout this paper. Let $G$ be a graph with vertex set $V(G)(|V(G)|=n)$ and edge set $E(G)$ $(|E(G)|=m)$. For a graph $G$, we let $d_{G}\left(v_{i}\right)$ be the degree of a vertex $v_{i}$ in $G$. The maximum vertex degree in $G$ is denoted by $\Delta$ and the minimum vertex degree by $\delta$. For each $v_{i} \in V(G)$, the set of neighbors of the vertex $v_{i}$ is denoted by $N_{G}\left(v_{i}\right)$. The irregularity index $t(G)$ of $G$ is defined as the number of distinct terms in the degree sequence of $G$ [1]. Denote by $p$, the number of pendent vertices in $G$. For terminology and notation not defined here, the reader is referred to Bondy and Murty [2].

Among the oldest and most studied topological indices, there are two classical vertex-degree based topological indices-the first Zagreb index and second Zagreb index. These two indices first appeared in [14], and were elaborated in [15]. Later they were used in the structure-property model (see [20]). The first Zagreb index $M_{1}(G)$ and the second Zagreb index $M_{2}(G)$ of a graph $G$ are defined, respectively, as

$$
M_{1}(G)=\sum_{v_{i} \in V(G)} d_{G}\left(v_{i}\right)^{2},
$$

and

$$
M_{2}(G)=\sum_{v_{i} v_{j} \in E(G)} d_{G}\left(v_{i}\right) d_{G}\left(v_{j}\right) .
$$

During the past decades, numerous results concerning Zagreb indices have been put forward, see $[3,5,7,9,13]$ and the references cited therein.

The Narumi-Katayama index of a graph $G$, denoted by $N K(G)$, is equal to the product of the degrees of the vertices of $G$, that is,

$$
N K(G)=\prod_{i=1}^{n} d_{G}\left(v_{i}\right) .
$$

Recently, the first and second multiplicative Zagreb indices [11, 18, 19] are defined as follows:

$$
\prod_{1}(G)=\prod_{i=1}^{n} d_{G}\left(v_{i}\right)^{2}
$$

and

$$
\prod_{2}(G)=\prod_{v_{i} v_{j} \in E(G)} d_{G}\left(v_{i}\right) d_{G}\left(v_{j}\right)=\prod_{i=1}^{n} d_{G}\left(v_{i}\right)^{d_{G}\left(v_{i}\right)} .
$$

The mathematical properties of the first and second multiplicative Zagreb indices have been given in $[6,11]$. 
$\mathrm{Xu}$ et al. recently defined the multiplicative Zagreb coindices as follows [21]:

$\overline{\prod_{1}}(G)=\prod_{v_{i} v_{j} \notin E(G)}\left(d_{G}\left(v_{i}\right)+d_{G}\left(v_{j}\right)\right)$ and $\overline{\prod_{2}}(G)=\prod_{v_{i} v_{j} \notin E(G)} d_{G}\left(v_{i}\right) d_{G}\left(v_{j}\right)$.

In the same reference, it has been also given the mathematical properties of the first and second multiplicative Zagreb coindices.

The paper is organized as follows. In Section 2, we give some lower and upper bounds on the Zagreb indices of graphs and trees, and characterize the extremal graphs. In Section 3, we obtain some lower and upper bounds on multiplicative Zagreb coindices of graphs and characterize the extremal graphs. In Section 4, we present some relations between first Zagreb index and Narumi-Katayama index, and (first and second) multiplicative Zagreb index and coindices of graphs.

\section{Bounds on the first Zagreb index of trees and graphs}

In this section we give some lower and upper bounds on the first Zagreb index of trees and graphs in terms of $n, t$ and $\Delta$. For this we need the following result:

Lemma 1. Let $T$ be a tree of order $n$ with irregularity index $t$. Then the number of pendent vertices in $T$ is

$$
p \geq \sum_{i=1}^{t-1}\left(d_{G}\left(v_{i}\right)-2\right)+2=\sum_{i=1}^{t-1} d_{G}\left(v_{i}\right)-2 t+4,
$$

where $d_{G}\left(v_{1}\right)>d_{G}\left(v_{2}\right)>\cdots>d_{G}\left(v_{t-1}\right)>d_{G}\left(v_{t}\right)=1$ and $d_{G}\left(v_{i}\right)$ is the degree of the vertex $v_{i}$ in $T$. Moreover, the equality holds in (1) if and only if $n=p+t-1$.

Proof. For any graph $G$, it is well known that $\sum_{i=1}^{n} d_{G}\left(v_{i}\right)=2 m$, where $m$ is the number of edges in $G$.

Since $T$ is a tree, we have $m=n-1$. Without loss of generality, we can assume that $d_{T}\left(v_{1}\right) \geq d_{T}\left(v_{2}\right) \geq \cdots \geq d_{T}\left(v_{n}\right)$. Let $p$ be the number of pendent vertices in $T$. For $i=n-p+1, n-p+2, \ldots, n$, we then have $d_{T}\left(v_{i}\right)=1$. 
Using these results, we get

$$
\begin{aligned}
& \sum_{i=1}^{n-p} d_{T}\left(v_{i}\right)+\sum_{i=n-p+1}^{n} d_{T}\left(v_{i}\right)=2(n-1) \Longleftrightarrow 2 n-p-2=\sum_{i=1}^{n-p} d_{T}\left(v_{i}\right) \\
& \Longleftrightarrow \quad p=\sum_{i=1}^{n-p}\left(d_{T}\left(v_{i}\right)-2\right)+2 \\
& \Longleftrightarrow \quad p \geq \sum_{i=1}^{t-1}\left(d_{T}\left(v_{i}\right)-2\right)+2 \quad \text { as } n-p \geq t-1 \text { and } d_{T}\left(v_{i}\right) \geq 2,
\end{aligned}
$$

where $i=1,2, \ldots, n-p$. Moreover, the equality holds in (2) if and only if $n=p+t-1$. Hence the equality holds in (1) if and only if $n=p+t-1$.

Let $\Gamma_{1}$ be the class of trees $T=(V, E)$ such that $T$ is a tree of order $n$, irregularity index $t$, maximum degree $\Delta$ and

$$
\Delta=t, \quad d_{T}\left(v_{i}\right)=1, i=t, t+1, \ldots, n .
$$

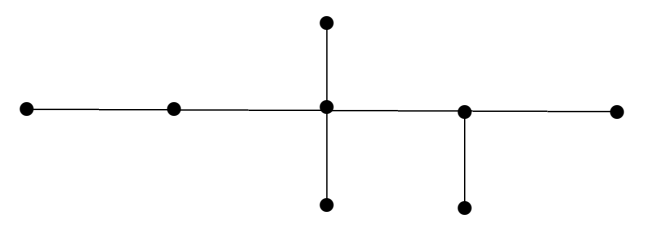

Figure 1:

Example 2. For a tree $T_{1}$ as in Figure 1 , it is clear that $n=8, \Delta=t=4$ and the number of pendent vertices are $5(=n-t+1)$. Moreover, the degree sequence of tree $T_{1}$ is $(4,3,2,1,1,1,1,1)$. Hence $T_{1} \in \Gamma$.

Now we are ready to give an upper bound on the first Zagreb index of trees in terms of $n, t$ and $\Delta$.

Theorem 3. Let $T$ be a tree of order $n$ with irregularity index $t$ and maximum degree $\Delta$. Then

$$
\begin{aligned}
M_{1}(T) \leq & \left(n-3-\frac{t(t-3)}{2}\right) \Delta^{2}-(t-1)(t-2) \Delta \\
& +\frac{1}{3}\left(t^{3}-3 t^{2}+2 t+6\right)
\end{aligned}
$$

with equality if and only if $G \in \Gamma_{1}$. 
Proof. Since the irregularity index of $T$ is $t$, let us consider a set $S(T)=$ $\left\{v_{1}, v_{2}, \ldots, v_{t-1}\right\}$ such that $\Delta=d_{G}\left(v_{1}\right)>d_{G}\left(v_{2}\right)>\ldots>d_{G}\left(v_{t-1}\right)>1$, where $d_{G}\left(v_{i}\right)$ is the degree of the vertex $v_{i}$ in $T$. Since $d_{G}\left(v_{i}\right) \leq \Delta$, for all $v_{i} \in V(T)$, we have

$$
\begin{aligned}
\sum_{v_{i} \in S(T)} d_{G}\left(v_{i}\right)^{2}=\sum_{i=1}^{t-1} d_{G}\left(v_{i}\right)^{2} \leq & \sum_{i=1}^{t-1}(\Delta-i+1)^{2} \\
= & \sum_{i=1}^{t-1}\left[(\Delta+1)^{2}+i^{2}-2 i(\Delta+1)\right] \\
= & (t-1)(\Delta+1)^{2}+\frac{1}{6}(t-1) t(2 t-1) \\
& -(\Delta+1) t(t-1) .
\end{aligned}
$$

Moreover, we have

$$
\sum_{v_{i} \in S(T)} d_{G}\left(v_{i}\right)=\sum_{i=1}^{t-1} d_{G}\left(v_{i}\right) \geq 2+3+\cdots+t=\frac{t(t+1)}{2}-1 .
$$

Let $p$ be the number of pendent vertices in $T$. Now,

$$
\begin{aligned}
M_{1}(T)= & \sum_{i=1}^{n} d_{G}\left(v_{i}\right)^{2} \\
= & \sum_{v_{i} \in V(T), d_{G}\left(v_{i}\right)=1} d_{G}\left(v_{i}\right)^{2}+\sum_{v_{i} \in S(T)} d_{G}\left(v_{i}\right)^{2} \\
& +\sum_{v_{i} \in V(T) \backslash S(T), d_{G}\left(v_{i}\right)>1} d_{G}\left(v_{i}\right)^{2} \\
\leq & p+\sum_{i=1}^{t-1} d_{G}\left(v_{i}\right)^{2}+(n-p-t+1) \Delta^{2} \quad \text { since } d_{G}\left(v_{i}\right) \leq \Delta \\
\leq & (n-t+1) \Delta^{2}-p\left(\Delta^{2}-1\right)+(t-1)(\Delta+1)^{2} \\
& +\frac{1}{6}(t-1) t(2 t-1)-(\Delta+1) t(t-1) \quad \text { by }(4) .
\end{aligned}
$$


By Lemma 1, the next step of last inequality is

$$
\begin{aligned}
\leq & n \Delta^{2}-\left(\sum_{i=1}^{t-1} d_{G}\left(v_{i}\right)-2 t+4\right)\left(\Delta^{2}-1\right)-\left(t^{2}-3 t+2\right) \Delta \\
& +\frac{1}{3} t^{3}-\frac{3}{2} t^{2}+\frac{13}{6} t-1 \\
\leq & n \Delta^{2}-\left(\frac{t(t-3)}{2}+3\right)\left(\Delta^{2}-1\right)-\left(t^{2}-3 t+2\right) \Delta+ \\
& \frac{1}{3} t^{3}-\frac{3}{2} t^{2}+\frac{13}{6} t-1 \text { by }(5),
\end{aligned}
$$

which gives the required result in (3). First part of the proof is completed.

Now suppose that the equality holds in (3). Then all the inequalities in the above must be equalities. From the equality in (7), we get

$$
n=p+t-1 \quad(\text { by Lemma } 1) .
$$

From the equality in (4), we get

$$
d_{G}\left(v_{i}\right)=\Delta-i+1, i=1,2, \ldots, t-1 .
$$

From the equality in (5), we get

$$
d_{G}\left(v_{i}\right)=t-i+1, \quad i=1,2, \ldots, t-1
$$

From the above results, we finally obtain $\Delta=t$ and $n=p+t-1$. Thus we have

$$
2 n-4=t(t-1), \Delta=t \quad \text { and } \quad d_{G}\left(v_{i}\right)=1 \text { for } i=t, t+1, \ldots, n .
$$

Hence $T \in \Gamma_{1}$.

Conversely, let $T \in \Gamma_{1}$. Then we have $\Delta=t, p=n-t+1$ and $n=$ $\frac{1}{2} t(t-1)+2$. We have

$$
M_{1}(T)=t^{2}+(t-1)^{2}+\cdots+2^{2}+1^{2}+(n-t)=\frac{1}{3} t^{3}+t^{2}-\frac{4}{3} t+2
$$

and

$$
\begin{aligned}
\left((n-3)-\frac{t(t-3)}{2}\right) \Delta^{2}- & (t-1)(t-2) \Delta+\frac{1}{3}\left(t^{3}-3 t^{2}+2 t+6\right)= \\
& \frac{1}{3} t^{3}+t^{2}-\frac{4}{3} t+2 .
\end{aligned}
$$

This completes the theorem. 
Let $\Gamma_{2}$ be the class of graphs $H_{1}=(V, E)$ such that $H_{1}$ is a graph of order $n$, irregularity index $t$, maximum degree $\Delta$ and

$$
\Delta=t, \quad d_{G}\left(v_{i}\right)=1, i=t+1, t+2, \ldots, n .
$$

Let $\Gamma_{3}$ be the class of graphs $H_{2}=(V, E)$ such that $H_{2}$ is a graph of order $n$, irregularity index $t$, maximum degree $\Delta$ and

$$
d_{G}\left(v_{i}\right)=\left\{\begin{array}{ll}
\Delta-i+1 & ; \quad i=1,2, \ldots, t \\
\Delta & ; \quad i=t+1, t+2, \ldots, n
\end{array} .\right.
$$

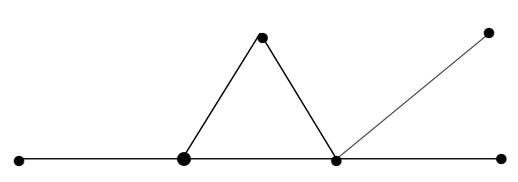

$G_{1}$

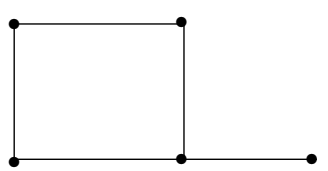

$G_{2}$

Figure 2:

Example 4. Two graphs $G_{1}$ and $G_{2}$ are depicted in Figure 2. For $G_{1}, n=6$, $\Delta=t=4$ and the number of pendent vertices are $3(=n-t+1)$. Moreover, the degree sequence of $G_{1}$ is $(4,3,2,1,1,1)$. Hence $G_{1} \in \Gamma_{2}$. For $G_{2}, n=5$, $\Delta=t=3$ and the number of maximum degree vertices are $3(=n-t+1)$. Moreover, the degree sequence of $G_{2}$ is $(3,3,3,2,1)$. Hence $G_{2} \in \Gamma_{3}$.

Now we give some lower and upper bounds on the first Zagreb index $M_{1}(G)$ of graphs $G$ in terms of $n, t$ and $\Delta$.

Theorem 5. Let $G$ be a graph of order $n$ with irregularity index $t$ and maximum degree $\Delta$. Then

$$
\text { and } \left.\begin{array}{rl}
M_{1}(G) \geq & \frac{1}{6} t(t+1)(2 t+1)+n-t \\
& M_{1}(G) \leq t(\Delta+1)^{2}+\frac{1}{6} t(t+1)(2 t+1) \\
& -(\Delta+1) t(t+1)+(n-t) \Delta^{2}
\end{array}\right\}
$$

with the lower and upper bounds in (8) become equality if and only if $G \in \Gamma_{2}$ and $G \in \Gamma_{3}$, respectively.

Proof. Since the irregularity index of $G$ is $t$, let us consider a set $S(G)=$ $\left\{v_{1}, v_{2}, \ldots, v_{t-1}\right\}$ such that $\Delta=d_{G}\left(v_{1}\right)>d_{G}\left(v_{2}\right)>\ldots>d_{G}\left(v_{t-1}\right)>1$, 
where $d_{G}\left(v_{i}\right)$ is the degree of the vertex $v_{i}$ in $G$. Since $\delta \leq d_{G}\left(v_{i}\right) \leq \Delta$, for all $v_{i} \in V(G)$, we have

$$
\begin{aligned}
M_{1}(G) & =\sum_{i=1}^{n} d_{G}\left(v_{i}\right)^{2} \\
& =\sum_{v_{i} \in S(G)} d_{G}\left(v_{i}\right)^{2}+\sum_{v_{i} \in V(G) \backslash S(G)} d_{G}\left(v_{i}\right)^{2} \\
& \geq \sum_{j=1}^{t} j^{2}+(n-t) 1=\frac{1}{6} t(t+1)(2 t+1)+n-t
\end{aligned}
$$

and

$$
\begin{aligned}
M_{1}(G) & =\sum_{v_{i} \in S(G)} d_{G}\left(v_{i}\right)^{2}+\sum_{v_{i} \in V(G) \backslash S(G)} d_{G}\left(v_{i}\right)^{2} \\
& \leq \sum_{j=1}^{t}(\Delta-j+1)^{2}+(n-t) \Delta^{2} \\
& =t(\Delta+1)^{2}+\frac{1}{6} t(t+1)(2 t+1)-(\Delta+1) t(t+1)+(n-t) \Delta^{2} .
\end{aligned}
$$

First part of the proof is done.

Now suppose that there exits an equality for the lower bound in (8). Then we must have

$\Delta=t, d_{G}\left(v_{i}\right)=t-i+1, i=1,2, \ldots, t-1$ and $d_{G}\left(v_{i}\right)=1, i=t, t+1, \ldots, n$.

Hence $T \in \Gamma_{2}$. On the other hand, if we suppose the existence of the equality for the upper bound in (8), then we must have

$$
d_{G}\left(v_{i}\right)=\Delta-i+1, i=1,2, \ldots, t \text { and } d_{G}\left(v_{i}\right)=\Delta, i=t+1, t+2, \ldots, n
$$

which implies $T \in \Gamma_{3}$.

Conversely, by taking $G \in \Gamma_{2}$ and $G \in \Gamma_{3}$ respectively, one can easily see that the truthness of the both equalities in (8).

\section{Bounds on the Multiplicative Zagreb coindices of graphs}

Let $\Gamma_{4}$ be the class of graphs $H_{4}=(V, E)$ such that $H_{4}$ is a connected graph of maximum degree $\Delta$, number of pendent vertices $p(p>0)$ and

$$
d_{H_{4}}\left(v_{1}\right)=d_{H_{4}}\left(v_{2}\right)=\cdots=d_{H_{4}}\left(v_{n-p}\right)=\Delta
$$


and

$$
d_{H_{4}}\left(v_{n-p+1}\right)=d_{H_{4}}\left(v_{n-p+2}\right)=\cdots=d_{H_{4}}\left(v_{n}\right)=1 \text {. }
$$

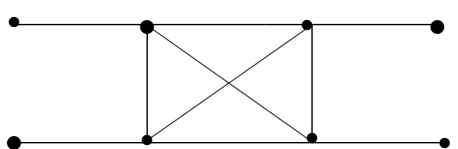

$G_{3}$

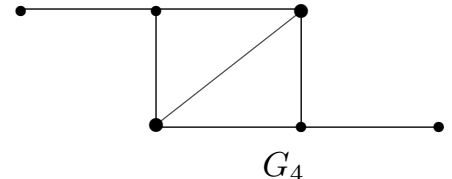

$G_{4}$

Figure 3:

Example 6. Let $G_{3}$ and $G_{4}$ be the graphs depicted in Figure 3. For $G_{3}, n=8$, $\Delta=4, t=2$ and the number of pendent vertices are $p=4$. Moreover, the degree sequence of graph $G_{3}$ is $(4,4,4,4,1,1,1,1)$. Hence $G_{3} \in \Gamma_{4}$. For $G_{4}$, $n=6, \Delta=3, t=2$ and the number of pendent vertices are $p=2$. Moreover, the degree sequence of $G_{4}$ is $(3,3,3,3,1,1)$. Hence $G_{4} \in \Gamma_{4}$.

Now we give some lower and upper bounds on the first multiplicative Zagreb coindex in terms of $n, m, p, \Delta$ and non-pendent minimum degree $\delta$.

Theorem 7. Let $G$ be a connected graph of order $n$ with $m$ edges, number of pendent vertices $p$, maximum degree $\Delta$ and non-pendent minimum degree $\delta$. Also, for simplicity, let $\mathcal{A}$ denotes $\frac{n(n-1)}{2}-m-\frac{p(p-1)}{2}-p(n-p-1)$. We then have

$$
\begin{aligned}
& \text { (i) } \bar{\Pi}_{1}(G) \geq 2^{\frac{p(p-1)}{2}}(\delta+1)^{p(n-p-1)}(2 \delta)^{\mathcal{A}}, \\
& \text { (ii) } \bar{\Pi}_{1}(G) \leq 2^{\frac{p(p-1)}{2}}(\Delta+1)^{p(n-p-1)}(2 \Delta)^{\mathcal{A}} .
\end{aligned}
$$

The equality holds in both (9) and (10) if and only if $G$ is isomorphic to a regular graph or $G \in \Gamma_{4}$.

Proof. The number of vertex pairs $\left(v_{i}, v_{j}\right)$ such that $v_{i} v_{j} \notin E(G)$ in $G$ is $\frac{n(n-1)}{2}-m$. Since $G$ is connected and the number of pendent vertices in $G$ is $p$, then $\frac{p(p-1)}{2}$ is the number of vertex pairs $\left(v_{i}, v_{j}\right)$ such that $v_{i} v_{j} \notin E(G)$ with $d_{G}\left(v_{i}\right)=d_{G}\left(v_{j}\right)=1, p(n-p-1)$ is the number of vertex pairs $\left(v_{i}, v_{j}\right)$ such that $v_{i} v_{j} \notin E(G)$ with $d_{G}\left(v_{i}\right)=1$ or $d_{G}\left(v_{j}\right)=1$, and $\frac{n(n-1)}{2}-$ $m-\frac{p(p-1)}{2}-p(n-p-1)$ is the number of vertex pairs $\left(v_{i}, v_{j}\right)$ such that 
$v_{i} v_{j} \notin E(G)$ with $d_{G}\left(v_{i}\right)>1$ and $d_{G}\left(v_{j}\right)>1$. From the above, we get

$$
\overline{\prod_{1}}(G)=\prod_{v_{i} v_{j} \notin E(G)}\left(d_{G}\left(v_{i}\right)+d_{G}\left(v_{j}\right)\right)
$$

which equals to

$$
\begin{aligned}
& \prod_{\substack{v_{i} v_{j} \notin E(G) \\
d_{G}\left(v_{i}\right)=1, d_{G}\left(v_{j}\right)=1}}\left(d_{G}\left(v_{i}\right)+d_{G}\left(v_{j}\right)\right) \\
& \times \prod_{\substack{v_{i} v_{j} \notin E(G) \\
d_{G}\left(v_{i}\right)>1, d_{G}\left(v_{j}\right)=1}}\left(d_{G}\left(v_{i}\right)+d_{G}\left(v_{j}\right)\right) \\
& \geq \\
& \geq \sum_{\substack{v_{i} v_{j} \notin E(G) \\
d_{G}\left(v_{i}\right)>1, d_{G}\left(v_{j}\right)>1}}\left(d_{G}\left(v_{i}\right)+d_{G}\left(v_{j}\right)\right) \\
& \frac{p(p-1)}{2}(\delta+1)^{p(n-p-1)}(2 \delta)^{\mathcal{A}},
\end{aligned}
$$

where $\mathcal{A}$ is defined as in the statement of the theorem. Moreover, the above equality holds if and only if $G$ is isomorphic to regular graph $(p=0)$ or $G \in \Gamma_{4}$ $(p>0)$.

Similarly, $\bar{\Pi}_{1}(G)$ equals to the

$$
\begin{aligned}
& \prod_{\substack{v_{i} v_{j} \notin E(G) \\
d_{G}\left(v_{i}\right)=1, d_{G}\left(v_{j}\right)=1}}\left(d_{G}\left(v_{i}\right)+d_{G}\left(v_{j}\right)\right) \\
\times & \prod_{\begin{array}{c}
v_{i} v_{j} \notin E(G) \\
d_{G}\left(v_{i}\right)>1, d_{G}\left(v_{j}\right)=1
\end{array}}\left(d_{G}\left(v_{i}\right)+d_{G}\left(v_{j}\right)\right) \\
& \prod_{\substack{v_{i} v_{j} \notin E(G) \\
d_{G}\left(v_{i}\right)>1, d_{G}\left(v_{j}\right)>1}}\left(d_{G}\left(v_{i}\right)+d_{G}\left(v_{j}\right)\right) \\
\leq & 2^{\frac{p(p-1)}{2}}(\Delta+1)^{p(n-p-1)}(2 \Delta)^{\mathcal{A}}
\end{aligned}
$$

where $\mathcal{A}$ is defined as in the statement of the theorem. We note that the last inequality becomes equality if and only if $G$ is isomorphic to regular graph $(p=0)$ or $G \in \Gamma_{4}(p>0)$.

Hence the result.

The following inequality was obtained by Furuichi [12].

Lemma 8. [12] For $a_{1}, a_{2}, \ldots, a_{n} \geq 0$ and $p_{1}, p_{2}, \ldots, p_{n} \geq 0$ satisfying

$$
\sum_{i=1}^{n} p_{i}=1
$$


there exists

$$
\sum_{i=1}^{n} p_{i} a_{i}-\prod_{i=1}^{n} a_{i}^{p_{i}} \geq n \lambda\left(\frac{1}{n} \sum_{i=1}^{n} a_{i}-\prod_{i=1}^{n} a_{i}^{1 / n}\right)
$$

where $\lambda=\min \left\{p_{1}, p_{2}, \ldots, p_{n}\right\}$. Moreover, equality in (11) holds if and only if $a_{1}=a_{2}=\cdots=a_{n}$.

Now we obtain an upper bound on $\bar{\Pi}_{2}(G)$ of graph $G$ in terms of $n, m, p$, the first Zagreb index $M_{1}(G)$ and the Narumi-Katayama index $N K(G)$.

Theorem 9. Let $G$ be a connected graph of order $n, m$ edges, $p$ pendent vertices and maximum degree $\Delta$. Then

$$
\begin{aligned}
\bar{\prod}_{2}(G) \leq & (N K(G))^{p}\left[\frac{(n-p-1)(2 m-p)+p-M_{1}(G)}{(n-p)(n-p-1)-2 m+p}\right. \\
& -\frac{(n-p)(n-\Delta-p-1)}{(n-p)(n-p-1)-2 m+p} \\
& \left.\times\left(\frac{2 m-p}{n-p}-(N K(G))^{1 /(n-p)}\right)\right]^{(n-p)(n-p-1)-2 m+p}
\end{aligned}
$$

Additionally, the equality holds in (12) if and only if $G \in \Gamma_{4}$ or $G$ is isomorphic to a regular graph.

Proof. Since $p$ is the number of pendent vertices in $G$, we need to consider the proof in two cases as in the following.

Case $(i)$ : The situation $p>0$ :

We can assume that

$$
d_{G}\left(v_{n-p+1}\right)=d_{G}\left(v_{n-p+2}\right)=\cdots=d_{G}\left(v_{n}\right)=1 .
$$

For $i=1,2, \ldots, n-p$, setting $a_{i}=d_{G}\left(v_{i}\right)$ and

$$
p_{i}=\frac{n-d_{G}\left(v_{i}\right)-p-1}{(n-p)(n-p-1)-2 m+p}
$$


in (11), we get

$$
\begin{gathered}
\sum_{i=1}^{n-p} \frac{d_{G}\left(v_{i}\right)\left(n-d_{G}\left(v_{i}\right)-p-1\right)}{(n-p)(n-p-1)-2 m+p}- \\
-\left(\prod_{i=1}^{n-p} d_{G}\left(v_{i}\right)^{n-d_{G}\left(v_{i}\right)-p-1}\right)^{\frac{1}{(n-p)(n-p-1)-2 m+p}} \geq \\
\geq \frac{(n-p)(n-\Delta-p-1)}{(n-p)(n-p-1)-2 m+p}\left(\frac{2 m-p}{n-p}-\left(\prod_{i=1}^{n-p} d_{G}\left(v_{i}\right)\right)^{1 /(n-p)}\right)
\end{gathered}
$$

that is,

$$
\begin{gathered}
\frac{(n-p-1)(2 m-p)+p-M_{1}(G)}{(n-p)(n-p-1)-2 m+p}- \\
-\left(\frac{\prod_{i=1}^{n-p} d_{G}\left(v_{i}\right)^{n-d_{G}\left(v_{i}\right)-1}}{\prod_{i=1}^{n-p} d_{G}\left(v_{i}\right)^{p}}\right)^{\frac{1}{(n-p)(n-p-1)-2 m+p}} \geq \\
\geq \frac{(n-p)(n-\Delta-p-1)}{(n-p)(n-p-1)-2 m+p}\left(\frac{2 m-p}{n-p}-\left(\prod_{i=1}^{n-p} d_{G}\left(v_{i}\right)\right)^{1 /(n-p)}\right) .
\end{gathered}
$$

After that, we actually have

$$
\begin{aligned}
\frac{\prod_{i=1}^{n-p} d_{G}\left(v_{i}\right)^{n-d_{G}\left(v_{i}\right)-1}}{\prod_{i=1}^{n-p} d_{G}\left(v_{i}\right)^{p}} & \leq\left[\frac{(n-p-1)(2 m-p)+p-M_{1}(G)}{(n-p)(n-p-1)-2 m+p}\right. \\
& -\frac{(n-p)(n-\Delta-p-1)}{(n-p)(n-p-1)-2 m+p} \\
& \left.\times\left(\frac{2 m-p}{n-p}-\left(\prod_{i=1}^{n-p} d_{G}\left(v_{i}\right)\right)^{1 /(n-p)}\right)\right]^{(n-p)(n-p-1)-2 m+p} .
\end{aligned}
$$

Using the above result with (13) and the definition of Narumi-Katayama index, we get

$$
\bar{\prod}_{2}(G)=\prod_{v_{i} v_{j} \notin E(G)} d_{G}\left(v_{i}\right) d_{G}\left(v_{j}\right)=\prod_{i=1}^{n} d_{G}\left(v_{i}\right)^{n-d_{G}\left(v_{i}\right)-1}
$$




$$
\begin{aligned}
\leq & (N K(G))^{p}\left[\frac{(n-p-1)(2 m-p)+p-M_{1}(G)}{(n-p)(n-p-1)-2 m+p}-\frac{(n-p)(n-\Delta-p-1)}{(n-p)(n-p-1)-2 m+p}\right. \\
& \left.\times\left(\frac{2 m-p}{n-p}-(N K(G))^{1 /(n-p)}\right)\right]^{(n-p)(n-p-1)-2 m+p}
\end{aligned}
$$

Case (ii) : The situation $p=0$ :

Similarly as in Case (i), for $i=1,2, \ldots, n$, setting $a_{i}=d_{G}\left(v_{i}\right)$ and $p_{i}=$ $\frac{n-d_{G}\left(v_{i}\right)-1}{n(n-1)-2 m}$ in (11), we get

$$
\begin{aligned}
\bar{\Pi}_{2}(G) \leq & {\left[\frac{2 m(n-1)-M_{1}(G)}{n(n-1)-2 m}\right.} \\
& \left.-\frac{n(n-\Delta-1)}{n(n-1)-2 m}\left(\frac{2 m}{n}-(N K(G))^{1 /(n)}\right)\right]^{n(n-1)-2 m} .
\end{aligned}
$$

Therefore the first part of the proof is completed.

Now let us suppose that the equality holds in (12). Then all the inequalities in the above must be equalities. For Case (i), we have $p>0$ and $d_{G}\left(v_{1}\right)=$ $d_{G}\left(v_{2}\right)=\cdots=d_{G}\left(v_{n-p}\right)$, by Lemma 8 . Hence $G \in \Gamma_{4}$. For Case (ii), we have $p=0$ and $d_{G}\left(v_{1}\right)=d_{G}\left(v_{2}\right)=\cdots=d_{G}\left(v_{n}\right)$, by Lemma 8 . Hence $G$ is isomorphic to a regular graph.

Conversely, let $G$ be isomorphic to a $r$-regular graph. Then $p=0,2 m=n r$, $M_{1}(G)=n r^{2}$ and $N K(G)=r^{n}$. We have

$\bar{\prod}_{2}(G)=r^{n(n-r-1)}=\left[\frac{(n-1) 2 m-n r^{2}}{n(n-1)-2 m}-\frac{n(n-\Delta-1)}{n(n-1)-2 m}\left(\frac{2 m}{n}-r\right)\right]^{n(n-1)-2 m}$.

Let $G \in \Gamma_{4}$. Then $2 m=(n-p) \Delta+p, M_{1}(G)=(n-p) \Delta^{2}+p$ and $N K(G)=\Delta^{n-p}$. Now we have

$$
\begin{array}{r}
(N K(G))^{p}\left[\frac{(n-p-1)(2 m-p)+p-M_{1}(G)}{(n-p)(n-p-1)-2 m+p}-\frac{(n-p)(n-\Delta-p-1)}{(n-p)(n-p-1)-2 m+p}\right. \\
\left.\quad \times\left(\frac{2 m-p}{n-p}-(N K(G))^{1 /(n-p)}\right)\right]^{(n-p)(n-p-1)-2 m+p} \\
=\Delta^{p(n-p)} \Delta^{(n-p)(n-p-\Delta-1)}=\Delta^{(n-p)(n-\Delta-1)}=\bar{\prod}_{2}(G) .
\end{array}
$$


This completes the proof.

\section{Some relations between first Zagreb index and Narumi- Katayama index, and multiplicative Zagreb index and coindices of graphs}

One of the present authors compared between various topological indices in his previous research works $[8,10,16]$. In this section we obtain some relations among topological indices of graphs. The first example comes to our mind is for star $K_{1, n-1}(n \geq 3)$,

$$
\prod_{2}\left(K_{1, n-1}\right)=(n-1)^{n-1}>1=\bar{\prod}_{2}\left(K_{1, n-1}\right),
$$

and the second example is for a cycle $C_{n}(n \geq 6)$,

$$
\prod_{2}\left(C_{n}\right)=2^{2 n}<2^{n(n-3)}=\bar{\prod}_{2}\left(C_{n}\right) .
$$

Now we can compare between the second multiplicative Zagreb index and the second multiplicative Zagreb coindex of graphs $G$.

Theorem 10. Let $G$ be a graph without isolated vertices of order $n$ with maximum degree $\Delta$ and minimum degree $\delta$. If $\delta \geq \frac{n-1}{2}$ ( or $\Delta \leq \frac{n-1}{2}$ ), then $\prod_{2}(G) \geq \bar{\prod}_{2}(G)\left(\right.$ or $\left.\prod_{2}(G) \leq \bar{\prod}_{2}(G)\right)$.

Proof. We clearly have

$$
\prod_{2}(G)=\prod_{v_{i} v_{j} \in E(G)} d_{G}\left(v_{i}\right) d_{G}\left(v_{j}\right)=\prod_{i=1}^{n} d_{G}\left(v_{i}\right)^{d_{G}\left(v_{i}\right)}
$$

and

$$
\bar{\prod}_{2}(G)=\prod_{v_{i} v_{j} \notin E(G)} d_{G}\left(v_{i}\right) d_{G}\left(v_{j}\right)=\prod_{i=1}^{n} d_{G}\left(v_{i}\right)^{n-d_{G}\left(v_{i}\right)-1} .
$$

From the above, we get

$$
\frac{\prod_{2}(G)}{\overline{\prod_{2}}(G)}=\prod_{i=1}^{n} d_{G}\left(v_{i}\right)^{2 d_{G}\left(v_{i}\right)-(n-1)} \geq 1 \text { as } d_{G}\left(v_{i}\right) \geq \delta \geq \frac{n-1}{2} .
$$

Similarly, one can easily prove that $\prod_{2}(G) \leq \overline{\prod_{2}}(G)$ if $\Delta \leq \frac{n-1}{2}$. 
For the isomorphism $G \cong K_{1, n-1}(n \geq 8)$, we have

$$
\begin{aligned}
\prod_{2}(G)+\bar{\prod}_{2}(G) & =(n-1)^{n-1}+1<(n-1)^{2}+2^{(n-1)(n-2) / 2} \\
& =\prod_{1}(G)+\bar{\prod}_{1}(G) .
\end{aligned}
$$

However we can present the following result:

Theorem 11. Let $G$ be a graph of order $n$. If $d_{G}\left(v_{i}\right) \geq 2$ for all $v_{i} \in V(G)$, then

$$
\prod_{2}(G)+\bar{\prod}_{2}(G) \geq \prod_{1}(G)+\bar{\prod}_{1}(G) .
$$

Proof. For each $v_{i} v_{j} \notin E(G)$, without loss of generality, we can assume that $d_{G}\left(v_{i}\right) \geq d_{G}\left(v_{j}\right)$. Thus we have

$$
d_{G}\left(v_{i}\right)\left(d_{G}\left(v_{j}\right)-1\right) \geq d_{G}\left(v_{j}\right) \text { as } d_{G}\left(v_{k}\right) \geq 2 \text { for all } v_{k} \in V(G),
$$

that is,

$$
d_{G}\left(v_{i}\right) d_{G}\left(v_{j}\right) \geq d_{G}\left(v_{i}\right)+d_{G}\left(v_{j}\right) \text { for } v_{i} v_{j} \notin E(G) .
$$

Using the above result, we get

$$
\begin{aligned}
\bar{\prod}_{2}(G)-\bar{\prod}_{1}(G) & =\prod_{v_{i} v_{j} \notin E(G)} d_{G}\left(v_{i}\right) d_{G}\left(v_{j}\right)-\prod_{v_{i} v_{j} \notin E(G)}\left(d_{G}\left(v_{i}\right)+d_{G}\left(v_{i}\right)\right) \\
& \geq 0
\end{aligned}
$$

and

$$
\begin{aligned}
\prod_{2}(G)-\prod_{1}(G) & =\prod_{v_{i} v_{j} \in E(G)} d_{G}\left(v_{i}\right) d_{G}\left(v_{j}\right)-\prod_{i=1}^{n} d_{G}\left(v_{i}\right)^{2} \\
& =\prod_{i=1}^{n} d_{G}\left(v_{i}\right)^{d_{G}\left(v_{i}\right)}-\prod_{i=1}^{n} d_{G}\left(v_{i}\right)^{2} \geq 0 \quad \text { as } d_{G}\left(v_{i}\right) \geq 2 .
\end{aligned}
$$

From the last two results, we get the required result.

Now we obtain the relation between the first multiplicative Zagreb coindex and the second multiplicative Zagreb coindex. Before, for simplicity, let us use the notation $\mathcal{A}$ for

$$
\frac{n(n-1)}{2}-m-\frac{p(p-1)}{2}-p(n-p-1)
$$

as in Theorem 7 . 
Theorem 12. Let $G$ be a connected graph of order $n$ with $m$ edges, number of pendent vertices $p$, maximum degree $\Delta$ and non-pendent minimum degree $\delta$. Then

$$
\text { (i) } \quad \bar{\prod}_{1}^{2}(G) \geq\left(\delta+\frac{1}{\delta}+2\right)^{p(n-p-1)} 4^{\mathcal{A}+\frac{p(p-1)}{2}} \overline{\prod_{2}}(G)
$$

with equality if and only if $G$ is isomorphic to a regular graph or $G \in \Gamma_{4}$, and

$$
\text { (ii) } \begin{aligned}
\bar{\prod}_{1}^{2}(G) \leq & 4^{\frac{p(p-1)}{2}\left(\frac{\Delta}{\delta}+\frac{\delta}{\Delta}+2\right)^{\mathcal{A}}} \\
& \times\left(\Delta+\frac{1}{\Delta}+2\right)^{p(n-p-1)} \overline{\prod_{2}(G)}
\end{aligned}
$$

with equality if and only if $G$ is isomorphic to a regular graph or $G \in \Gamma_{4}$.

Proof. For $d_{G}\left(v_{i}\right), d_{G}\left(v_{j}\right)>1$, since $\frac{\Delta}{\delta} \geq \frac{d_{G}\left(v_{i}\right)}{d_{G}\left(v_{j}\right)} \geq \frac{\delta}{\Delta}$, by [4], we have

$$
\begin{aligned}
4 \leq\left(\sqrt{\frac{d_{G}\left(v_{i}\right)}{d_{G}\left(v_{j}\right)}}-\sqrt{\frac{d_{G}\left(v_{j}\right)}{d_{G}\left(v_{i}\right)}}\right)^{2}+4= & \left(\sqrt{\frac{d_{G}\left(v_{i}\right)}{d_{G}\left(v_{j}\right)}}+\sqrt{\frac{d_{G}\left(v_{j}\right)}{d_{G}\left(v_{i}\right)}}\right)^{2} \leq \\
& \leq\left(\sqrt{\frac{\Delta}{\delta}}+\sqrt{\frac{\delta}{\Delta}}\right)^{2}
\end{aligned}
$$

with equality holding if and only if $d_{G}\left(v_{i}\right)=\Delta, d_{G}\left(v_{j}\right)=\delta$ for any $v_{i} v_{j} \notin E(G)$ and $d_{G}\left(v_{i}\right) \geq d_{G}\left(v_{j}\right)$.

In the proof of Theorem 7 , we mentioned that $\frac{p(p-1)}{2}$ is the number of vertex pairs $\left(v_{i}, v_{j}\right)$ such that $v_{i} v_{j} \notin E(G)$ with $d_{G}\left(v_{i}\right)=d_{G}\left(v_{j}\right)=1$, $p(n-p-1)$ is the number of vertex pairs $\left(v_{i}, v_{j}\right)$ such that $v_{i} v_{j} \notin E(G)$ with $d_{G}\left(v_{i}\right)=1$ or $d_{G}\left(v_{j}\right)=1$, and $\frac{n(n-1)}{2}-m-\frac{p(p-1)}{2}-p(n-p-1)$ is the number of vertex pairs $\left(v_{i}, v_{j}\right)$ such that $v_{i} v_{j} \notin E(G)$ with $d_{G}\left(v_{i}\right)>1$ and $d_{G}\left(v_{j}\right)>1$.

Using the above result with (18), we get

$$
\begin{aligned}
\prod_{\substack{v_{i} v_{j} \notin E(G) \\
d_{G}\left(v_{i}\right)>1, d_{G}\left(v_{j}\right)>1}}\left(\frac{d_{G}\left(v_{i}\right)}{d_{G}\left(v_{j}\right)}+\frac{d_{G}\left(v_{j}\right)}{d_{G}\left(v_{i}\right)}+2\right) & \leq \prod_{v_{i} v_{j} \notin E(G)}\left(\frac{\Delta}{\delta}+\frac{\delta}{\Delta}+2\right) \\
& =\left(\frac{\Delta}{\delta}+\frac{\delta}{\Delta}+2\right)^{\mathcal{A}}
\end{aligned}
$$


and

$$
\prod_{\substack{v_{i} v_{j} \notin E(G) \\ d_{G}\left(v_{i}\right)>1, d_{G}\left(v_{j}\right)>1}}\left(\frac{d_{G}\left(v_{i}\right)}{d_{G}\left(v_{j}\right)}+\frac{d_{G}\left(v_{j}\right)}{d_{G}\left(v_{i}\right)}+2\right) \geq 4^{\mathcal{A}} .
$$

Moreover, the above two equalities hold if and only if $d_{G}\left(v_{i}\right)=d_{G}\left(v_{j}\right)$ for all $v_{i}$ and $v_{j}$ such that $d_{G}\left(v_{i}\right)>1, d_{G}\left(v_{j}\right)>1$.

Now we have

$$
\prod_{\substack{v_{i} v_{j} \notin E(G) \\ G\left(v_{i}\right)=1, d_{G}\left(v_{j}\right)=1}}\left(\frac{d_{G}\left(v_{i}\right)}{d_{G}\left(v_{j}\right)}+\frac{d_{G}\left(v_{j}\right)}{d_{G}\left(v_{i}\right)}+2\right)=4^{\frac{p(p-1)}{2}}
$$

and

$$
\begin{aligned}
\prod_{\substack{v_{i} v_{j} \notin E(G) \\
d_{G}\left(v_{i}\right)>1, d_{G}\left(v_{j}\right)=1}}\left(\frac{d_{G}\left(v_{i}\right)}{d_{G}\left(v_{j}\right)}+\frac{d_{G}\left(v_{j}\right)}{d_{G}\left(v_{i}\right)}+2\right) & =\prod_{v_{i} v_{j} \notin E(G)}\left(d_{G}\left(v_{i}\right)+\frac{1}{d_{G}\left(v_{i}\right)}+2\right) \\
& \geq\left(\delta+\frac{1}{\delta}+2\right)^{p(n-p-1)}
\end{aligned}
$$

with equality if and only if $d_{G}\left(v_{i}\right)=\delta$ for all $v_{i}$ such that $d_{G}\left(v_{i}\right)>1$.

Also we have

$$
\begin{aligned}
\prod_{\substack{v_{i} v_{j} \notin E(G) \\
d_{G}\left(v_{i}\right)>1, d_{G}\left(v_{j}\right)=1}}\left(\frac{d_{G}\left(v_{i}\right)}{d_{G}\left(v_{j}\right)}+\frac{d_{G}\left(v_{j}\right)}{d_{G}\left(v_{i}\right)}+2\right) & =\prod_{v_{i} v_{j} \notin E(G)}\left(d_{G}\left(v_{i}\right)+\frac{1}{d_{G}\left(v_{i}\right)}+2\right) \\
& \leq\left(\Delta+\frac{1}{\Delta}+2\right)^{p(n-p-1)}
\end{aligned}
$$

with equality if and only if $d_{G}\left(v_{i}\right)=\Delta$ for all $v_{i}$.

By the definition, since we have

$$
\bar{\prod}_{1}(G)=\prod_{v_{i} v_{j} \notin E(G)}\left(d_{G}\left(v_{i}\right)+d_{G}\left(v_{j}\right)\right) \text { and } \overline{\prod_{2}}(G)=\prod_{v_{i} v_{j} \notin E(G)} d_{G}\left(v_{i}\right) d_{G}\left(v_{j}\right),
$$


we get

$$
\begin{aligned}
& \frac{\bar{\prod}_{1}^{2}(G)}{\bar{\prod}_{2}(G)}=\prod_{v_{i} v_{j} \notin E(G)} \frac{\left(d_{G}\left(v_{i}\right)+d_{G}\left(v_{j}\right)\right)^{2}}{d_{G}\left(v_{i}\right) d_{G}\left(v_{j}\right)} \\
& =\prod_{\substack{v_{i} v_{j} \notin E(G) \\
d_{G}\left(v_{i}\right)=1, d_{G}\left(v_{j}\right)=1}}\left(\frac{d_{G}\left(v_{i}\right)}{d_{G}\left(v_{j}\right)}+\frac{d_{G}\left(v_{j}\right)}{d_{G}\left(v_{i}\right)}+2\right) \prod_{\substack{v_{i} v_{j} \notin E(G) \\
d_{G}\left(v_{i}\right)>1, d_{G}\left(v_{j}\right)=1}}\left(\frac{d_{G}\left(v_{i}\right)}{d_{G}\left(v_{j}\right)}+\frac{d_{G}\left(v_{j}\right)}{d_{G}\left(v_{i}\right)}+2\right) \\
& \times \prod_{v_{i} v_{j} \notin E(G)}\left(\frac{d_{G}\left(v_{i}\right)}{d_{G}\left(v_{j}\right)}+\frac{d_{G}\left(v_{j}\right)}{d_{G}\left(v_{i}\right)}+2\right) \\
& d_{G}\left(v_{i}\right)>1, d_{G}\left(v_{j}\right)>1 \\
& \geq\left(\delta+\frac{1}{\delta}+2\right)^{p(n-p-1)} 4^{\left[\frac{n(n-1)}{2}-m-p(n-p-1)\right] .}
\end{aligned}
$$

Moreover, the above equality holds if and only if $G$ is isomorphic to regular graph $(p=0)$ or $G \in \Gamma_{4}(p>0)$.

Similarly,

$$
\begin{aligned}
& \frac{\bar{\Pi}_{1}^{2}(G)}{\bar{\prod}_{2}(G)}=\prod_{\substack{v_{i} v_{j} \notin E(G) \\
d_{G}\left(v_{i}\right)=1, d_{G}\left(v_{j}\right)=1}}\left(\frac{d_{G}\left(v_{i}\right)}{d_{G}\left(v_{j}\right)}+\frac{d_{G}\left(v_{j}\right)}{d_{G}\left(v_{i}\right)}+2\right) \prod_{\substack{v_{i} v_{j} \notin E(G) \\
d_{G}\left(v_{i}\right)>1, d_{G}\left(v_{j}\right)=1}}\left(\frac{d_{G}\left(v_{i}\right)}{d_{G}\left(v_{j}\right)}+\frac{d_{G}\left(v_{j}\right)}{d_{G}\left(v_{i}\right)}+2\right) \\
& \times \prod_{\substack{v_{i} v_{j} \notin E(G) \\
d_{G}\left(v_{i}\right)>1, d_{G}\left(v_{j}\right)>1}}\left(\frac{d_{G}\left(v_{i}\right)}{d_{G}\left(v_{j}\right)}+\frac{d_{G}\left(v_{j}\right)}{d_{G}\left(v_{i}\right)}+2\right) \\
& \leq 4^{\frac{p(p-1)}{2}}\left(\Delta+\frac{1}{\Delta}+2\right)^{p(n-p-1)}\left(\frac{\Delta}{\delta}+\frac{\delta}{\Delta}\right)^{\mathcal{A}} .
\end{aligned}
$$

Moreover, the above equality holds if and only if $G$ is isomorphic to regular graph $(p=0)$ or $G \in \Gamma_{4}(p>0)$. This completes the proof.

Similarly, we obtain another relation between the first multiplicative Zagreb coindex and second multiplicative Zagreb coindex. To do that, again let us use the notation $\mathcal{A}$ for the algebraic statement $\frac{n(n-1)}{2}-m-\frac{p(p-1)}{2}-p(n-$ $p-1)$. 
Theorem 13. Let $G$ be a connected graph of order $n$ with $m$ edges, number of pendent vertices $p$, maximum degree $\Delta$ and non-pendent minimum degree $\delta$. Then

$$
\text { (i) } \begin{aligned}
\bar{\prod}_{1}(G) & \geq 2^{\frac{p(p-1)}{2}}\left(\frac{2}{\Delta}\right)^{\mathcal{A}} \\
\times & \left(\frac{1}{\Delta}+1\right)^{p(n-p-1)} \bar{\prod}_{2}(G)
\end{aligned}
$$

with equality if and only if $G$ is isomorphic to a regular graph or $G \in \Gamma_{4}$, and

$$
\text { (ii) } \begin{aligned}
\bar{\prod}_{1}(G) & \leq 2^{\frac{p(p-1)}{2}}\left(\frac{2}{\delta}\right)^{\mathcal{A}} \\
& \times\left(\frac{1}{\delta}+1\right)^{p(n-p-1)} \bar{\prod}_{2}(G)
\end{aligned}
$$

with equality if and only if $G$ is isomorphic to a regular graph or $G \in \Gamma_{4}$.

Proof. We have

$$
\begin{gathered}
\frac{\bar{\prod}_{1}(G)}{\bar{\Pi}_{2}(G)}=\prod_{v_{i} v_{j} \notin E(G)}\left(\frac{1}{d_{G}\left(v_{i}\right)}+\frac{1}{d_{G}\left(v_{j}\right)}\right) \\
=\prod_{\substack{v_{i} v_{j} \notin E(G) \\
d_{G}\left(v_{i}\right)=1, d_{G}\left(v_{j}\right)=1}}\left(\frac{1}{d_{G}\left(v_{i}\right)}+\frac{1}{d_{G}\left(v_{j}\right)}\right) \prod_{\substack{v_{i} v_{j} \notin E(G) \\
d_{G}\left(v_{i}\right)>1, d_{G}\left(v_{j}\right)=1}}\left(\frac{1}{d_{G}\left(v_{i}\right)}+\frac{1}{d_{G}\left(v_{j}\right)}\right) \\
\times \prod_{\substack{v_{i} v_{j} \notin E(G) \\
d_{G}\left(v_{i}\right)>1, d_{G}\left(v_{j}\right)>1}}\left(\frac{1}{d_{G}\left(v_{i}\right)}+\frac{1}{d_{G}\left(v_{j}\right)}\right) .
\end{gathered}
$$

We have $\delta \leq d_{G}\left(v_{i}\right) \leq \Delta$ for non-pendent vertices. For $v_{i} v_{j} \notin E(G)$ with $d_{G}\left(v_{i}\right)>1, d_{G}\left(v_{j}\right)>1$,

$$
\frac{2}{\Delta} \leq \frac{1}{d_{G}\left(v_{i}\right)}+\frac{1}{d_{G}\left(v_{j}\right)} \leq \frac{2}{\delta}
$$

and for $v_{i} v_{j} \notin E(G)$ with $d_{G}\left(v_{i}\right)>1, d_{G}\left(v_{j}\right)=1$,

$$
1+\frac{1}{\Delta} \leq \frac{1}{d_{G}\left(v_{i}\right)}+\frac{1}{d_{G}\left(v_{j}\right)} \leq 1+\frac{1}{\delta} .
$$


Using the same technique as in Theorem 12, we get the required result in (19) and (20). Moreover, the equality holds in (19) and (20) if and only if $G$ is isomorphic to regular graph $(p=0)$ or $G \in \Gamma_{4}(p>0)$. This completes the proof.

Lemma 14. [17] Let $x_{1}, x_{2}, \ldots, x_{N}$ be non negative numbers, and let

$$
\alpha=\frac{1}{N} \sum_{i=1}^{N} x_{i} \quad \text { and } \quad \gamma=\left(\prod_{i=1}^{N} x_{i}\right)^{1 / N}
$$

be their arithmetic and geometric means, respectively. Then

$$
\frac{1}{N(N-1)} \sum_{i<j}\left(\sqrt{x_{i}}-\sqrt{x_{j}}\right)^{2} \leq \alpha-\gamma \leq \frac{1}{N} \sum_{i<j}\left(\sqrt{x_{i}}-\sqrt{x_{j}}\right)^{2} .
$$

Moreover, equality holds if and only if $x_{1}=x_{2}=\cdots=x_{n}$.

Let $\Gamma_{5}$ be the class of graphs $H_{5}=(V, E)$ such that $H_{5}$ is a graph of order $n$ with maximum degree $\Delta$, minimum degree $\delta$ and

$$
\Delta \neq \delta, \quad d_{H_{5}}\left(v_{2}\right)=d_{H_{5}}\left(v_{3}\right)=\cdots=d_{H_{5}}\left(v_{n-1}\right) .
$$

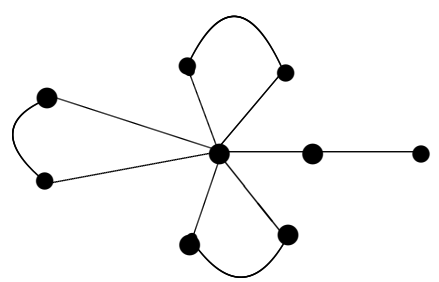

$G_{5}$

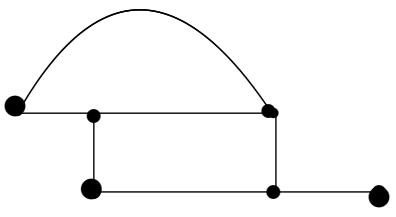

$G_{6}$

Figure 4:

Example 15. Let $G_{5}$ and $G_{6}$ be the graphs depicted in Figure 4. For $G_{5}$, $n=9, \Delta=7, \delta=1$ and $t=3$. Moreover, the degree sequence of graph $G_{5}$ is $(7,2,2,2,2,2,2,2,1)$. Hence $G_{5} \in \Gamma_{5}$. For $G_{6}, n=6, \Delta=3, \delta=1$ and $t=2$. Moreover, the degree sequence of $G_{4}$ is $(3,3,3,3,3,1)$. Hence $G_{6} \in \Gamma_{5}$.

Now we obtain a relation between the first Zagreb index and NarumiKatayama index of graph $G$. 
Theorem 16. Let $G$ be a graph of order $n$ with $m$ edges, maximum degree $\Delta$ and minimum degree $\delta$. Then

$$
\begin{aligned}
(n-2)(N K(G))^{2 /(n-2)} \geq & (\Delta \delta)^{2 /(n-2)}\left[(n-3)\left(\Delta^{2}+\delta^{2}\right)\right. \\
& \left.+(2 m-\Delta-\delta)^{2}-(n-3) M_{1}(G)\right]
\end{aligned}
$$

and

$$
\begin{array}{r}
(\Delta \delta)^{2 /(n-2)}\left[(2 m-\Delta-\delta)^{2}+\left(\Delta^{2}+\delta^{2}\right)-M_{1}(G)\right] \geq \\
(n-2)(n-3)(N K(G))^{2 /(n-2)} .
\end{array}
$$

Moreover, both the above equalities hold if and only if $G$ is isomorphic to a regular graph or $G \in \Gamma_{5}$.

Proof. Setting in Lemma $14, N=n-2$ and $x_{i}=d_{G}\left(v_{i}\right)^{2}, i=2,3, \ldots, n-1$, we get

$$
\begin{aligned}
& \alpha=\frac{1}{N} \sum_{i=1}^{N} x_{i}=\frac{1}{n-2} \sum_{i=2}^{n-1} d_{G}\left(v_{i}\right)^{2}=\frac{1}{n-2}\left(M_{1}(G)-\Delta^{2}-\delta^{2}\right) \\
& \gamma=\left(\prod_{i=1}^{N} x_{i}\right)^{1 / N}=\left(\prod_{i=2}^{n-1} d_{G}\left(v_{i}\right)^{2}\right)^{1 /(n-2)}=\left(\frac{\prod_{i=1}^{n} d_{G}\left(v_{i}\right)}{\Delta \delta}\right)^{2 /(n-2)} \\
& =\frac{(N K(G))^{2 /(n-2)}}{(\Delta \delta)^{2 /(n-2)}}
\end{aligned}
$$

and

$$
\begin{aligned}
\sum_{2 \leq i<j \leq n-1} & \left(\sqrt{x_{i}}-\sqrt{x_{j}}\right)^{2}=\sum_{2 \leq i<j \leq n-1}\left(d_{G}\left(v_{i}\right)-d_{G}\left(v_{j}\right)\right)^{2} \\
& =(n-3) \sum_{i=2}^{n-1} d_{G}\left(v_{i}\right)^{2}-2 \sum_{2 \leq i<j \leq n-1} d_{G}\left(v_{i}\right) d_{G}\left(v_{j}\right) \\
& =(n-3)\left(M_{1}(G)-\Delta^{2}-\delta^{2}\right)-\sum_{i=2}^{n-1} d_{G}\left(v_{i}\right)\left(2 m-d_{G}\left(v_{i}\right)-\Delta-\delta\right) \\
& =(n-2) M_{1}(G)-(n-2)\left(\Delta^{2}+\delta^{2}\right)-(2 m-\Delta-\delta)^{2} .
\end{aligned}
$$


Using the above results in Lemma 14, we get

$$
\begin{gathered}
\frac{(n-2) M_{1}(G)-(n-2)\left(\Delta^{2}+\delta^{2}\right)-(2 m-\Delta-\delta)^{2}}{(n-2)(n-3)} \\
\leq \frac{M_{1}(G)-\Delta^{2}-\delta^{2}}{n-2}-\frac{(N K(G))^{2 /(n-2)}}{(\Delta \delta)^{2 /(n-2)}} \\
\leq \frac{(n-2) M_{1}(G)-(n-2)\left(\Delta^{2}+\delta^{2}\right)-(2 m-\Delta-\delta)^{2}}{n-2} \&,,
\end{gathered}
$$

that is,

$$
\begin{gathered}
(n-2)(N K(G))^{2 /(n-2)} \\
\geq(\Delta \delta)^{2 /(n-2)}\left[(n-3)\left(\Delta^{2}+\delta^{2}\right)+(2 m-\Delta-\delta)^{2}-(n-3) M_{1}(G)\right]
\end{gathered}
$$

and

$$
\begin{gathered}
(\Delta \delta)^{2 /(n-2)}\left[(2 m-\Delta-\delta)^{2}+\left(\Delta^{2}+\delta^{2}\right)-M_{1}(G)\right] \\
\geq(n-2)(n-3)(N K(G))^{2 /(n-2)} .
\end{gathered}
$$

By Lemma 14, one can see easily that both the equality holds in (23) if and only if $d_{G}\left(v_{2}\right)=d_{G}\left(v_{3}\right)=\cdots=d_{G}\left(v_{n-1}\right)$. First part of the proof is done.

First suppose that the equality holds in (21). Then $d_{G}\left(v_{2}\right)=d_{G}\left(v_{3}\right)=$ $\cdots=d_{G}\left(v_{n-1}\right)$. If $\Delta=\delta$, then $G$ is isomorphic to a regular graph. Otherwise, $\Delta \neq \delta$ and hence $G \in \Gamma_{5}$. Conversely, one can see easily that the equality holds in (21) for regular graph.

Let $G \in \Gamma_{5}$. Then we have $\Delta \neq \delta$ and $d_{G}\left(v_{2}\right)=d_{G}\left(v_{3}\right)=\cdots=$ $d_{G}\left(v_{n-1}\right)=d$, (say). Now,

$$
\begin{aligned}
& (n-2)(N K(G))^{2 /(n-2)}=(n-2) d^{2}(\Delta \delta)^{2 /(n-2)}, \\
& (n-2)(n-3)(N K(G))^{2 /(n-2)}=(n-3)(n-2) d^{2}(\Delta \delta)^{2 /(n-2)}, \\
& (\Delta \delta)^{2 /(n-2)}\left[(2 m-\Delta-\delta)^{2}+\left(\Delta^{2}+\delta^{2}\right)-M_{1}(G)\right]=(n-3)(n-2) d^{2}(\Delta \delta)^{2 /(n-2)}, \\
& \quad \text { and }(\Delta \delta)^{2 /(n-2)}\left[(n-3)\left(\Delta^{2}+\delta^{2}\right)+(2 m-\Delta-\delta)^{2}-(n-3) M_{1}(G)\right] \\
& \quad=(\Delta \delta)^{2 /(n-2)}\left[(n-2)^{2} d^{2}-(n-3)(n-2) d^{2}\right]=(n-2) d^{2}(\Delta \delta)^{2 /(n-2)} .
\end{aligned}
$$

Hence the equality holds in (21).

Similarly, we can show that the equality holds in (22) if and only if $G$ is isomorphic to a regular graph or $G \in \Gamma_{5}$.

Acknowledgement. The first author is supported by the National Research Foundation funded by the Korean government with the grant no. 
2013R1A1A2009341. The other authors are partially supported by Research Project Offices of N. Erbakan, Uludag and Selcuk Universities. This paper has been prepared during the Kinkar Ch. Das's visit in Turkey that was partially funded by TUBITAK 2221-Programme. The fifth author is supported by Uludag University Research Fund, Project Nos: F-2015/23 and F-2015/17.

\section{References}

[1] N. Akgüneş, A. S. Çevik, A new bound of radius with irregularity index, Applied Mathematics and Computation 219 (11) (2013) 5750-5753.

[2] J. A. Bondy, U. S. R. Murty, Graph Theory with Applications, Macmillan London and Elsevier, New York, 1976.

[3] K. C. Das, Maximizing the sum of the squares of the degrees of a graph, Discrete Math. 285 (2004) 57-66.

[4] K. C. Das, On geometrical-arithmetic index of graphs, MATCH Commun. Math. Comput. Chem. 64 (3) (2010) 619-630.

[5] K. C. Das, On comparing Zagreb indices of graphs, MATCH Commun. Math. Comput. Chem. 63 (2) (2010) 433-440.

[6] K. C. Das, A. Yurttaş, M. Togan, I. N. Cangül, A. S. Çevik, The multiplicative Zagreb indices of graph operations, Journal of Inequalities and Applications, 2013, 2013: 90.

[7] K. C. Das, I. Gutman, B. Zhou, New upper bounds on Zagreb indices, J. Math. Chem. 46 (2009) 514-521.

[8] K. C. Das, N. Trinajstić, Relationship between the eccentric connectivity index and Zagreb indices, Comput. Math. Appl. 62 (2011) 1758-1764.

[9] K. C. Das, I. Gutman, B. Horoldagva, Comparison between Zagreb indices and Zagreb coindices, MATCH Commun. Math. Comput. Chem. 68 (1) (2012) 189-198.

[10] K. C. Das, N. Trinajstić, Comparison between geometric-arithmetic indices, Croat. Chem. Acta 85 (3) (2012) 353-357.

[11] M. Eliasi, A. Iranmanesh, I. Gutman, Multiplicative versions of first Zagreb index, MATCH Commun. Math. Comput. Chem. 68 (2012) 217-230.

[12] S. Furuichi, On refined Young inequalities and reverse inequalities, J. Math. Ineq. 5 (2011) 21-31.

[13] B. Horoldagva, K. C. Das, On comparing Zagreb indices of graphs, Hacettepe Journal of Mathematics and Statistics 41 (2) (2012) 223-230. 
[14] I. Gutman, N. Trinajstić, Graph theory and molecular orbitals. Total $\pi$ electron energy of alternate hydrocarbons, Chem. Phys. Lett. 17 (1972) $535-538$.

[15] I. Gutman, B. Ruščić, N. Trinajstić, C. F. Wilcox, Graph theorey and molecular orbitals. XII. Acyclic polyenes, J. Chem. Phys. 62 (1975) 3399.

[16] H. Hua, K. C. Das, The relationship between eccentric connectivity index and Zagreb indices, Discrete Applied Math. 161 (2013) 2480-2491.

[17] H. Kober, On the arithmetic and geometric means and the Hölder inequality, Proc. Am. Math. Soc. 59 (1958) 452-459.

[18] R. Todeschini, D. Ballabio, V. Consonni, Novel molecular descriptors based on functions of new vertex degrees [in:] Novel molecular structure descriptors- Theory and applications I, I. Gutman, B. Furtula, (eds.), pp. 73-100. Univ. Kragujevac, Kragujevac, 2010.

[19] R. Todeschini, V. Consonni, New local vertex invariants and molecular descriptors based on functions of the vertex degrees, MATCH Commun. Math. Comput. Chem. 64 (2010) 359-372.

[20] R. Todeschini, V. Consonni, Handbook of Molecular Descriptors, WileyVCH, Weinheim, 2000.

[21] K. Xu, K. C. Das, K. Tang, On the multiplicative Zagreb coindex of graphs, Opuscula Math. 33 (1) (2013) 191-204.

Kinkar Ch. DAS,

Department of Mathematics,

Sungkyunkwan University,

Suwon 440-746, Republic of Korea.

Email: kinkardas2003@googlemail.com

Nihat AKGUNES,

Department of Mathematics-Computer Sciences,

Necmettin Erbakan University, Faculty of Science,

Meram Yeniyol, 42100, Konya, Turkey.

Email: nakgunes@konya.edu.tr

Muge TOGAN, Aysun YURTTAS, Ismail Naci CANGUL

Department of Mathematics,

Uludag University, Faculty of Science and Art,

Gorukle Campus, 16059, Bursa, Turkey.

Emails: mugecapkin@yahoo.com.tr, aysunyurttas@gmail.com,cangul@uludag.edu.tr

Ahmet Sinan CEVIK,

Department of Mathematics,

Selcuk University, Faculty of Science,

Campus, 42075, Konya, Turkey.

Email: sinan.cevik@selcuk.edu.tr 